\title{
Robust One-Class Kernel Spectral Regression
}

\author{
Shervin Rahimzadeh Arashloo ${ }^{(}$and Josef Kittler ${ }^{(}$, Life Member, IEEE
}

\begin{abstract}
The kernel null-space technique is known to be an effective one-class classification (OCC) technique. Nevertheless, the applicability of this method is limited due to its susceptibility to possible training data corruption and the inability to rank training observations according to their conformity with the model. This article addresses these shortcomings by regularizing the solution of the null-space kernel Fisher methodology in the context of its regression-based formulation. In this respect, first, the effect of the Tikhonov regularization in the Hilbert space is analyzed, where the one-class learning problem in the presence of contamination in the training set is posed as a sensitivity analysis problem. Next, the effect of the sparsity of the solution is studied. For both alternative regularization schemes, iterative algorithms are proposed which recursively update label confidences. Through extensive experiments, the proposed methodology is found to enhance robustness against contamination in the training set compared with the baseline kernel null-space method, as well as other existing approaches in the OCC paradigm, while providing the functionality to rank training samples effectively.
\end{abstract}

Index Terms-Contamination, kernel null-space technique, one-class classification (OCC), regression, regularization.

\section{INTRODUCTION}

W HILE for a wide variety of pattern classification problems, there is an abundance of data for the system design, there are applications where the quantity of the training data is severely limited, or the data are in some respect unrepresentative or imbalanced [1]. This is manifest in applications where the cost associated with collecting samples from a specific class is prohibitively high, or samples take unexpected forms at the test time. There also exist other scenarios where the recognition task is inherently open, leading to inaccurately defined class(es). In these situations, a particular class may not be very well represented by the available training samples or be totally void of samples, causing a degradation in the performance of conventional multiclass/binary-class classifiers.

Manuscript received March 24, 2019; revised November 10, 2019 and February 26, 2020; accepted March 5, 2020. Date of publication April 2, 2020; date of current version March 1, 2021. This work was supported in part by the Engineering and Physical Sciences Research Council (EPSRC) Program under Grant (FACER2VM) EP/N007743/1 and in part by the EPSRC/Defence Science and Technology Laboratory (DSTL)/Multidisciplinary University Research Initiatives (MURI) under Project EP/R018456/1. (Corresponding author: Shervin Rahimzadeh Arashloo.)

Shervin Rahimzadeh Arashloo is with the Department of Computer Engineering, Faculty of Engineering, Bilkent University, 06800 Ankara, Turkey (e-mail: s.rahimzadeh@cs.bilkent.edu.tr).

Josef Kittler is with the Centre for Vision, Speech and Signal Processing (CVSSP), Department of Electronic Engineering, Faculty of Engineering and Physical Sciences, University of Surrey, Guildford GU2 7XH, U.K. (e-mail: J.kittler@ surrey.ac.uk).

This article has supplementary downloadable material available at https://ieeexplore.ieee.org, provided by the authors.

Color versions of one or more of the figures in this article are available online at https://ieeexplore.ieee.org.

Digital Object Identifier 10.1109/TNNLS.2020.2979823
An alternative and more effective approach in such cases is offered by the one-class classification (OCC) paradigm [2]. OCC aims to identify patterns from the target class, conforming to a specific behavior, and distinguish them from nontarget objects. It differs from the conventional multiclass/binary-class formulation in that it primarily uses samples from a single class for training. More concretely, assume $X=\left\{x_{1}, \ldots, x_{n}\right\}$ to be a set of observations where $x_{i} \in \mathbb{R}^{d}$ is a realization of a multivariate random variable $x$ characterized by the target probability density function $p(x)$. One-class learning tries to specify the support domain of $p(x)$ using a one-class classifier $h(z)$ as

$$
h(z)=\lceil f(z) \geq \tau\rceil= \begin{cases}1, & z \in \mathcal{T} \\ 0, & \text { otherwise }\end{cases}
$$

where $\mathcal{T}$ denotes the target class, and the function $f($.) encodes the similarity of an observation $z$ to $\mathcal{T}$, while $\lceil$.$\rceil stands for the Iverson brackets. Parameter \tau$ controls the fraction of observations that lie within the support domain of the target distribution.

OCC is typically believed to be a more difficult problem than its multiclass/binary-class counterpart due to the lack of nontarget training samples, impeding the estimation of a decision boundary between the target and nontarget observations. Yet, it forms the basis of a variety of different applications, including intrusion detection [3], novelty detection [4], fault detection in safety-critical systems [5], fraud detection [6], insurance [7], health care [8], and surveillance [9].

In order to identify a pattern as normal or novel, a one-class classifier is trained on a training set consisting of (typically) normal samples and then used to gauge the similarity of a test sample to those previously observed in the training set. In this case, the generalization capability of the one-class learner on new data plays a central role. In a real-life one-class learning problem, however, not all observations in the training set would conform to the model equally. In practice, the training set may be corrupted and incorporate noisy or nontarget observations that may degrade performance, emphasizing the requirement for mechanisms to identify such samples. The identification of the degree of normality of samples in a given set is also useful in its own right for specific applications, such as ranking and retrieving items from a database for a given query, pruning contaminated data sets, as well as in decision threshold setting. It also opens the possibility of using the detected nontarget samples to refine the decision boundary. Consequently, a ranking of the set of training observations according to their degree of fit with the model is highly desirable in a one-class learning paradigm. 
While there exist a variety of different OCC methods and fine-grained categorisation of such methods exists [10]-[12], they can be roughly identified as either generative or nongenerative [13]. Generative approaches include a model for data generation, whereas nongenerative methods lack a transparent link to the data. Among the well-known instances of the generative approaches are the parametric and nonparametric density estimation methods [14]-[16], neuralnetwork-based approaches [17], [18], sparse representation methods [19], [20], and so on. Notable representatives of nongenerative methods include support vector-based approaches [21], [22], convex hull methods [23], [24], cluster approaches [25], subspace techniques [26]-[30], or others [31]. As a result of the emphasis put on classification, rather than on generative process modeling, the nongenerative methods are typically believed to yield better classification performance.

Among other nongenerative methods, the kernel null-space approach is known to be a very successful methodology for OCC. In particular, it has been found to perform better than many state-of-the-art OCC techniques by exploiting a nonlinear projection function corresponding to the Fisher criterion for classification [28], [32]-[35]. The null-space approach has also been employed for classification purposes other than OCC [36], [37]. More specifically, in this methodology, a discriminative feature subspace is inferred such that observations from a specific class are mapped onto a single point, resulting in a zero within-class scatter. On the other hand, a positive between-class scatter is obtained since any other sample is mapped onto another distinct point in the feature subspace.

Although the superiority of this approach over some other alternatives has been confirmed in different studies [28], [32], [33], nevertheless, in practice, it suffers from a number of limitations. In the one-class learning paradigm, it is natural to expect that not all training samples equally adhere to the model inferred. In extreme cases, a fraction of training observations may be corrupted or even counterexamples. As such, it is desirable to rank the training observations according to their conformity with the inferred model. A ranking of the training samples may then allow one to enhance the performance by discarding noisy observations from the training set or using incompatible samples as counterexamples to refine the decision boundaries and thus enhance the robustness against contamination in the training set. In addition, the ranking of the training data also facilitates setting an appropriate decision threshold for the system to achieve (approximately) a desired error rate on the test set. Furthermore, there exist applications where one is only interested in ranking observations in a given data set in a completely unsupervised fashion. However, as in the kernel null-space methodology, all training samples corresponding to a particular class are projected onto the same point in the optimal feature subspace, and there exists no straightforward mechanism to gauge the compatibility of (and subsequently rank) training samples with the underlying model. The sensitivity of the kernel null-space technique to noisy observations in the training set and their propensity to seriously degrade its performance in practical settings will be demonstrated in Section V.

\section{A. Overview of the Proposed Approach}

This article addresses the aforementioned limitations of the kernel null-space methodology by: 1) allowing the labels of observations to vary and, thus, in contrast to fixed hard labels to provide soft confidences so as to facilitate ranking of the training data; 2) studying the effect of regularization on the regression framework and its ability to deal with training set contamination; and 3) refining the decision boundary of a one-class classifier by automatically detecting outliers in the training set and utilizing them as counterexamples. Regarding the first contribution, an alternating direction minimization approach is proposed where the discriminant function in the Hilbert space and label confidences are optimized concurrently. The second contribution is achieved by introducing two alternative regularization techniques, either based on the Tikhonov regularization or on sparsity, which are shown to improve the one-class kernel system robustness against contaminations in the training set. Finally, in terms of the third contribution, a variant of the proposed approach is presented, which makes use of any detected nontarget samples in the training set to refine the decision boundary and enhance the system performance.

\section{B. Outline of This Article}

The rest of this article is organized as follows. In Section II, a review of related work is provided. A brief overview of the kernel null-space technique and its regression-based formulation is presented in Section III. The proposed robust kernel spectral regression approach is introduced in Section IV. The results of an experimental evaluation of the proposed methodology are presented and discussed in Section V. Finally, conclusions are drawn in Section VI.

\section{RELATED WORK}

There exists a variety of different approaches to the OCC problem. From a classification perspective, these methods may be categorized as the density methods, the boundary methods, and the reconstruction-based techniques [11]. The density-based approaches try to estimate the density of the training data followed by setting a threshold on the estimated density. For this purpose, several different distributions have been assumed in practice, including the Gaussian or a Poisson distribution. Example methods in this category of OCC methods include [38]-[40]. The second group of OCC approaches is considered to be reconstruction-based methods. Although the methods in this category are designed to model the data as their primary objective, yet, in practice, they may be applied to the OCC problem. In this category of methods, typically, a model is chosen and fit to the data, which makes it viable to represent new objects in terms of their affinity to the generative model. Modeling necessitates some prior knowledge and assumptions to be made regarding the data and the underlying generative process. For model fitting purposes, typically, a set of prototypes or subspaces is chosen followed by a minimization of the reconstruction error. Prominent examples of this category of approaches are the k-means clustering [41], learning vector quantization [42], 
self-organizing maps, PCA, a mixture of PCAs, Diabolo networks, and autoencoders [43], [43].

The third category of OCC techniques is that of boundary methods. This methodology is fuelled, in part, by the observation that solving the more general problem of characterizing the generative process for the data or estimating a density in an OCC problem might seem too demanding when for classification purposes, only the data boundary is required. Consequently, in this category of approaches, the goal is to optimize a boundary encompassing the target set. Sample methods falling into this category include the k-centers [25], NN-d [11], the SVDD [21], and so on. The proposed approach in this article sits in the third group of the organizational framework, i.e., the boundary-based category. However, as the proposed approach relies on a Rayleigh quotient formulation, a brief overview of the methods most similar to the proposed approach, specifically those operating on a Rayleigh quotient criterion, is provided in the following. As one of the main objectives of this study is to provide a robust OCC approach, a brief review of the prominent approaches for robust unsupervised OCC will be provided too. For a more detailed review of the OCC problem, one may consult [10]-[12].

Similar to the kernel null-space methodology, there exist other approaches operating on a Rayleigh quotient criterion. As an instance of the unsupervised methods in this category, the work in [16] utilizes kernel PCA for novelty detection where the reconstruction residual of a test sample with respect to the inferred subspace is considered as a novelty measure. Other work [44] proposes a strategy to improve the convergence of the kernel algorithm based on an iterative kernel PCA. A robustified PCA that deals with outliers in the training set is proposed in [45].

A one-class kernel Fisher discriminant classifier is presented in [26] and [27], which is based on the idea of separating the data from their negatively replicated counterparts and involves an eigenvalue decomposition of the kernel matrix. In this approach, once the data are mapped onto the feature space, a Mahalanobis distance to the mean of the fit Gaussian is used as a test statistic. As indicated in [27], for kernel maps projecting input data into a higher-dimensional space, the Gaussianity assumption for the target data may not hold in general. An increasing deviation from normality may render the results of the method presented in [26] and [27] unreliable. Another work in [28] proposed a Fisher-based null space method where all training samples of one class are projected onto a single point. The proposed method treats multiple known classes jointly and detects novel instances with respect to the set of classes using a single model operating on a joint subspace where the training samples of all known classes are presumed to have zero variance. In a follow-up work [46], it is proposed to incorporate locality in the null space approach of [28] by considering only the most similar patterns to a query sample. In [33], an incremental version of the method in [28] is proposed to enhance computational efficiency.

A generalized Rayleigh quotient specifically designed for outlier detection is presented in [29] and [47], where the method tries to find an optimal hyperplane that is closest to the target data and farthest from the outliers utilizing two scatter matrices corresponding to the outliers and target data. In [47], the generalized eigenvalue problem is replaced by an approximate conjugate gradient solution to moderate the computational cost of the method in [29]. A later study [48] tries to address the limitations of the method in [29] and [47] in terms of the availability of outlier samples and a difference in the densities of target and nontarget observations via the null-space approach.

While the majority of existing work on OCC based on the Rayleigh quotient formulation requires computationally intensive eigendecomposition of large matrices, the work in [32] presents a one-class approach that replaces costly eigenanalysis computations by a regression-based formulation [49]. Among other unsupervised novelty detection techniques, the dual-principal-component pursuit (DPCP) approach [50] learns a linear subspace from data corrupted by outliers using nonconvex $l_{1}$ optimization. It is shown that DPCP can tolerate as many outliers as the square of the number of inliers, thus improving upon other robust PCA methods. Another method, known as outlier pursuit (OP), is an efficient convex optimization-based algorithm [51] to perform a robust principal component analysis that, under mild assumptions on the uncorrupted points, recovers the exact optimal lowdimensional subspace and identifies the corrupted points. The fast median subspace (FMS) method is a nonconvex robust subspace recovery approach [52], designed to be least affected by corruptions in the training set. It has been demonstrated to converge to close vicinity of the correct subspace in few iterations. A leading unsupervised technique, among others, is that of Self Representation-based Outlier detection (SRO) [53]. It obtains a weighted directed graph, defines a Markov Chain via self-representation, and identifies outliers via random walks.

\section{BACKGROUND}

A brief overview of the kernel Fisher null-space approach [28], [32], [33] and its regression-based formulation [32] is provided in the following.

\section{A. Null-Space Fisher Analysis}

In statistical pattern classification, a widely used design objective is the Fisher criterion where one tries to infer a projection function from the input space into a feature space such that the within-class scatter of the data associated with each class is minimized while maximizing the between-class scatter. More specifically, in a Fisher classifier, the aim is to maximize the criterion function $\mathbf{J}(\varphi)$

$$
\underset{\varphi}{\arg \max } \mathbf{J}(\varphi)=\underset{\varphi}{\arg \max } \frac{\varphi^{\top} \mathbf{S}_{\mathbf{b}} \varphi}{\varphi^{\top} \mathbf{S}_{\mathbf{w}} \varphi}
$$

where $\mathbf{S}_{\mathbf{b}}$ denotes the between-class scatter matrix, $\mathbf{S}_{\mathbf{w}}$ denotes the within-class scatter matrix, and $\varphi$ is a basis defining one axis of the subspace.

In an OCC problem and the absence of nontarget training observations, the origin may be used as a counterexample. With regards to the Fisher criterion, a theoretically optimal projection is the one yielding a zero within-class scatter while 
providing a positive between-class scatter, referred to as a null projection [28], [32], [33]. Thus, in a null-space Fisher classifier

$$
\begin{gathered}
\varphi^{\top} \mathbf{S}_{\mathbf{w}} \varphi=0 \\
\varphi^{\top} \mathbf{S}_{\mathbf{b}} \varphi>0 .
\end{gathered}
$$

A null projection function attains the optimum of $\mathbf{J}(\varphi)$ in (2) and, thus, provides the best separability with respect to the Fisher criterion. It can be shown that one may compute at most $C-1$ null projection directions, with $C$ being the number of classes. In a one-class formulation, since target observations are assumed to form a single class, while (hypothetical) nontarget samples correspond to a second class, only a single optimizer for (2), given as the eigenvector corresponding to the largest eigenvalue of the following eigenproblem, exists:

$$
\mathbf{S}_{\mathbf{b}} \varphi=\lambda \mathbf{S}_{\mathbf{w}} \varphi .
$$

Once the null projection direction is determined, the projection of a sample $x$ onto the null-space (hereafter referred to as response) is found as

$$
\mathbf{y}=\varphi^{\top} \mathbf{x}
$$

In order to handle data with inherently nonlinear structure, nonlinear (kernel) extensions of this methodology are proposed [28], [32], [33]. In kernel methods, a kernel function is utilized to implicitly project the data into a high dimensional space, known as the reproducing kernel Hilbert space (RKHS) in an attempt to make the data more easily separable in this new space. These methods typically require eigendecompositions of dense matrices.

\section{B. One-Class Kernel Spectral Regression}

As eigencomputations associated with the kernel nullspace technique are computationally demanding, an alternative approach based on spectral regression [called one-class kernel spectral regression (OC-KSR)] was proposed in [32], operating on an equivalent representation of the Fisher criterion (see the Supplementary Material). The OC-KSR method is based on two principles: 1) in order for the within-class scatter to be zero while achieving a positive between-class scatter, the observations corresponding to a specific class must be mapped onto the same point in the optimal feature subspace, distinct from all other classes, i.e., the responses (elements of $\mathbf{y}$ ) corresponding to samples of a particular class must be equal, while being different from those corresponding to other class(es) and 2) in an RKHS, the function realizing the abovementioned projection from the input space onto a feature subspace can be represented in terms of real numbers $\alpha_{i}$ 's and a positive semidefinite kernel function $\kappa(.,$.$) as [30]$

$$
f(.)=\sum_{i=1}^{n} \alpha_{i} \kappa\left(., x_{i}\right)
$$

where $x_{i}$ 's denote training observations. In the OC-KSR approach [32], finding the optimal coefficients $\alpha_{i}$ 's is then posed as a regression problem

$$
f(.)^{o p t}=\underset{f(.)}{\arg \min }\left\{\sum_{i=1}^{n}\left(f\left(x_{i}\right)-y_{i}\right)^{2}+\delta\|f\|_{K}^{2}\right\}
$$

or equivalently as

$$
\boldsymbol{\alpha}^{\mathrm{opt}}=\underset{\boldsymbol{\alpha}}{\arg \min }\left\{\|\mathbf{K} \boldsymbol{\alpha}-\mathbf{y}\|^{2}+\delta \boldsymbol{\alpha}^{\top} \mathbf{K} \boldsymbol{\alpha}\right\}
$$

where $\delta$ is a regularization parameter and $\|\cdot\|_{K}^{2}$ denotes the norm in the RKHS, while $\mathbf{K}$ stands for the kernel matrix. The optimal solution $\boldsymbol{\alpha}$ to the abovementioned problem satisfies

$$
\left(\mathbf{K}+\delta \mathbf{I}_{\mathbf{n}}\right) \boldsymbol{\alpha}=\mathbf{y}
$$

where $\mathbf{I}_{\mathbf{n}}$ denotes an identity matrix of size $n$. Very often, selecting the most appropriate kernel function depends on the problem at hand. Different alternatives in this respect include linear, polynomial, RBF, sigmoid, and so on. While the linear kernel may be considered as the computationally most efficient kernel, in practice, the RBF may be considered as one of the most widely used kernel functions. In order to compensate for the lack of a priori knowledge regarding the selection of an appropriate kernel function, some research has been conducted to learn the kernel from the data. Notable examples of this category of methods include those which try to model the kernel as a linear convex combination of multiple base kernels and then try to optimize the parameters for such a linear combination [54], [55].

In the OC-KSR method of [32], the kernel function is an RBF, and thus, the kernel matrix is positive definite. As the kernel matrix in the OC-KSR approach is invertible, no regularization is imposed (i.e., the regularization parameter $\delta$ is set to zero) on $\boldsymbol{\alpha}$. Based on the availability of training data, two cases are considered in the OC-KSR method: if only positive training samples are available, the target response vector $\mathbf{y}$ is shown to be $\mathbf{y}=(\overbrace{1, \ldots, 1}^{n})^{\top}$. When both positive and negative training observations are available, the target response vector $\mathbf{y}$ is given as $\mathbf{y}=(\overbrace{1, \ldots, 1}^{n-n_{o}}, \overbrace{0, \ldots, 0}^{n_{0}})^{\top}$ (up to a scale factor) where without loss of generality, it is assumed that the nontarget instances are collected as the last $n_{0}$ samples of the training set. Given $\mathbf{y}$, solving (9) for $\boldsymbol{\alpha}$ is performed efficiently using the Cholesky factorization via Sherman's march algorithm [56]. Once $\boldsymbol{\alpha}$ is determined, a test sample may be projected onto the null feature subspace using (6). In the decision-making stage, the (Euclidean) distance between the projection of a test sample and that of the target class is used as a dissimilarity criterion.

\section{Robust One-Class Kernel Spectral Regression}

In this section, the proposed approach to build a robust oneclass classifier based on the kernel null-space methodology in the presence of contamination in the training set is presented. In the proposed method, starting from the given labels of the training samples, label confidences are updated iteratively, and soft labels are assigned to the training observations, reflecting their degree of normality. As a result, although the proposed method is designed to detect novelty with respect to a given set of training samples, nevertheless, it can be directly used for observation ranking purposes in an unsupervised setting as will be discussed in the following. 
As noted earlier, the proposed approach is based on a regularized regression formulation, that is

$$
P(\boldsymbol{\alpha}, \mathbf{y})=\|\mathbf{K} \boldsymbol{\alpha}-\mathbf{y}\|^{2}+\mathcal{R}(\boldsymbol{\alpha})
$$

where $\mathbf{y}$ is the expected responses for observations and $\mathcal{R}(\boldsymbol{\alpha})$ encodes a desired regularization of the solution $\boldsymbol{\alpha}$. The regularization, in general, may serve different purposes. First, when the number of variables exceeds the number of observations, the least-squares problem is ill-posed, and it is, therefore, impossible to solve the associated optimization problem as infinitely many solutions exist. Regularization, in this case, allows the introduction of additional constraints that help to uniquely determine the solution. The second scenario, where regularization may be deployed, corresponds to the case where the number of variables does not exceed the number of samples, but the model learned suffers from poor generalization capability. In such cases, regularization is used to improve the generalization performance of the model by constraining it during the training phase. The regularization term, thus, imposes a limitation on the function space available by introducing a penalty to avoid certain regions of the function space. In this work, given sparse and noisy samples of a function $f($.$) from a corrupted data set, regularization$ constraints the function and maintains a tradeoff between data fidelity and some constraints on the solution function. The imposed constraint may, for example, enforce the solution to be sparse or reflect other prior knowledge about the solution, such as constraining its norm in the corresponding space. In such cases, regularization methods typically correspond to priors on the solution to a least-squares problem.

Following a regularized regression formulation, the proposed approach to handle contaminations in the training set is to optimize the objective function $P$ not only with respect to $\boldsymbol{\alpha}$ but also with respect to $\mathbf{y}$. While optimization with respect to $\alpha$ is the standard approach to specify the parameters characterizing the projection function given by (6), optimization with respect to $\mathbf{y}$ reflects (and compensates for) the absence of prior knowledge regarding the conformity of individual observations to the model. This is fundamentally different from the ordinary OC-KSR method [32] as well as other flavors of the kernel null-space technique [28], [33], where $\mathbf{y}$ is fixed and $\boldsymbol{\alpha}$ optimizers of $P$ are sought. Moreover, the method in [32] does not impose a regularization on the model when deriving the final solution (i.e., $\delta$ is set to 0 ). In essence, given an initial guess regarding object labels (i.e., the responses $\mathbf{y}$ ), the proposed approach updates label confidences and derives soft responses for all training samples while, at the same time, optimizing the objective function with respect to $\boldsymbol{\alpha}$. However, by allowing $\mathbf{y}$ to vary, the solution to (9) is undefined. Hence, another constraint is required to prevent $\boldsymbol{\alpha}$ going to zero. Imposing a fixed $L_{2}$-norm would prevent the solution to degenerate. Note that rescaling $\boldsymbol{\alpha}$ only introduces a scaling effect on the responses $\mathbf{y}=\mathbf{K} \boldsymbol{\alpha}$ and, consequently, would not adversely affect the classification performance. The optimization is realized via a block coordinate descent minimization approach, alternating between minimizing $P$ with respect to $\alpha$ and minimizing it with respect to $\mathbf{y}$.

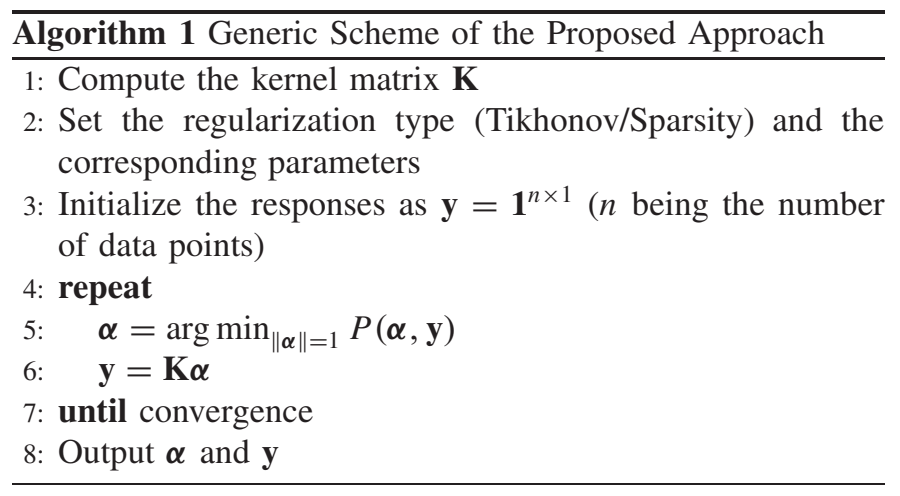

Optimizing $P$ with respect to $\mathbf{y}$ can be realized by setting its partial derivative with respect to $\mathbf{y}$ equal to zero, that is

$$
\frac{\partial P}{\partial \mathbf{y}}=-2(\mathbf{K} \boldsymbol{\alpha}-\mathbf{y})=0
$$

which gives $\mathbf{y}=\mathbf{K} \boldsymbol{\alpha}$. As such, the generic scheme of the proposed approach can be summarized as Algorithm 1. It can be shown that (under some conditions set out in the Supplementary Material) the alternating minimization scheme of Algorithm 1 is convergent. That is, by denoting $\boldsymbol{\alpha}_{t+1}=$ $\arg \min _{\|\boldsymbol{\alpha}\|=1} P\left(\boldsymbol{\alpha}, \mathbf{y}_{t}\right)$ and $\mathbf{y}_{t+1}=\arg \min _{\mathbf{y}} P\left(\boldsymbol{\alpha}_{t+1}, \mathbf{y}\right)$ for iteration $t+1$, one has

$$
P\left(\boldsymbol{\alpha}_{t}, \mathbf{y}_{t}\right) \geq P\left(\boldsymbol{\alpha}_{t+1}, \mathbf{y}_{t}\right) \geq P\left(\boldsymbol{\alpha}_{t+1}, \mathbf{y}_{t+1}\right) .
$$

The regularization types considered in this work are those of Tikhonov and sparsity. As a result, the objective function $P(\boldsymbol{\alpha}, \mathbf{y})$ is bounded from below, and thus, the nonincreasing sequence $\lim _{t \rightarrow \infty} P\left(\boldsymbol{\alpha}_{t}, \mathbf{y}_{t}\right)$ is convergent.

Solving the minimization problem $\boldsymbol{\alpha}^{\text {opt }}=\arg \min _{\|\boldsymbol{\alpha}\|=1}$ $P(\boldsymbol{\alpha}, \mathbf{y})$ is dependent upon the specific regularization imposed on the solution. Although other possibilities exist, in this work, two commonly used regularization schemes of Tikhonov and sparsity are considered for $\mathcal{R}(\boldsymbol{\alpha})$, discussed in the following.

\section{A. Tikhonov Regularization}

In the case of a Tikhonov regularization (also known as ridge regression), $\mathcal{R}(\boldsymbol{\alpha})=\delta \boldsymbol{\alpha}^{\top} \mathbf{K} \boldsymbol{\alpha}$, and the objective function $P(\boldsymbol{\alpha}, \mathbf{y})$ would be

$$
P(\boldsymbol{\alpha}, \mathbf{y})=\|\mathbf{K} \boldsymbol{\alpha}-\mathbf{y}\|^{2}+\delta \boldsymbol{\alpha}^{\top} \mathbf{K} \boldsymbol{\alpha} .
$$

In this case, the problem $\boldsymbol{\alpha}^{o p t}=\arg \min _{\|\boldsymbol{\alpha}\|=1} P(\boldsymbol{\alpha}, \mathbf{y})$ can be solved by setting the partial derivative of $P(\alpha, \mathbf{y})$ with respect to $\alpha$ to zero, that is

$$
\frac{\partial P}{\partial \boldsymbol{\alpha}}=2 \mathbf{K}(\mathbf{K} \boldsymbol{\alpha}-\mathbf{y})+2 \delta \mathbf{K} \boldsymbol{\alpha}=0
$$

which gives

$$
\boldsymbol{\alpha}^{o p t}=\left(\mathbf{K}+\delta \mathbf{I}_{n}\right)^{-1} \mathbf{y} .
$$

Combining the two equations for $\boldsymbol{\alpha}$ and $\mathbf{y}$, the proposed approach based on a Tikhonov regularization is given as Algorithm 2. In a way, the Tikhonov regularization favors models that provide predictions that are as smooth functions of the data as possible. In other words, such a regularization 


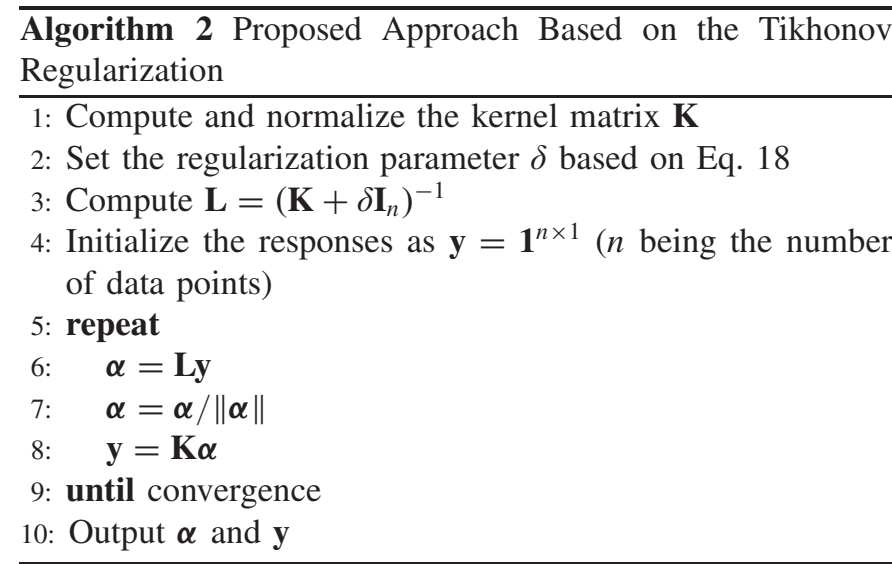

scheme penalizes the coefficients of larger magnitude, thereby producing a more parsimonious solution, incorporating a set of coefficients with smaller variance, which is particularly advantageous when making an inference in a noisy data set.

1) Optimal Regularization Parameter: Algorithm 2 provides a procedure to find the optimal parameters $\boldsymbol{\alpha}$ and $\mathbf{y}$. Yet, it does not specify how to choose the Tikhonov regularization parameter $\delta$. In order to infer the optimal Tikhonov regularization parameter, the one-class learning problem in the presence of outliers is posed as a sensitivity analysis problem in this work. In this respect, the optimal Tikhonov regularization parameter is derived so as to minimize the sensitivity of the solution $\boldsymbol{\alpha}$ with respect to contaminations in the training set. For this purpose, let us consider a set of contaminated observations $X=\left\{x_{1}, \ldots, x_{n}\right\}$ for which the true labels are recorded in vector $\mathbf{y}$. It is assumed that we are not informed of the true labels in the contaminated data set. Instead, an initial guess for object labels may be assumed. In the absence of any prior knowledge, the initial assumption for all observations (recorded in vector $\mathbf{y}^{\prime}$ ) may be that of being target samples, i.e., $\mathbf{y}^{\prime}=\{\overbrace{1, \ldots, 1}^{n}\}$. $\mathbf{y}^{\prime}$ corresponds to the noisy assumption of labels, which deviates from the true labels by $\Delta \mathbf{y}$, i.e., $\mathbf{y}^{\prime}=\mathbf{y}+\Delta \mathbf{y}$. Using the assumed noisy labels $\mathbf{y}^{\prime}$, the weight vector $\boldsymbol{\alpha}^{\prime}$ for the Tikhonov regularized problem is given as $\boldsymbol{\alpha}^{\prime}=(\mathbf{K}+\delta I)^{-1} \mathbf{y}^{\prime}$. In practice, it is desirable to have $\boldsymbol{\alpha}^{\prime}$ as close as possible to the true $\boldsymbol{\alpha}$ (derived based on $\mathbf{y}$ ) so that the responses obtained as $\mathbf{K} \boldsymbol{\alpha}^{\prime}$ are close to the true responses $\mathbf{y}=\mathbf{K} \boldsymbol{\alpha}$. This is essentially a sensitivity analysis problem where one is given a perturbed response vector $\mathbf{y}^{\prime}=\mathbf{y}+\Delta \mathbf{y}$, and the goal is to find a solution $\boldsymbol{\alpha}^{\prime}$ with a minimal difference from the ideal solution $\boldsymbol{\alpha}$. Assuming $\left\|\boldsymbol{\alpha}^{\prime}\right\|>0$, the sensitivity of the solution with respect to perturbations in object labels is defined as

$$
S=\frac{\left\|\boldsymbol{\alpha}^{\prime}-\boldsymbol{\alpha}\right\|}{\left\|\boldsymbol{\alpha}^{\prime}\right\|} .
$$

Apparently, minimizing the sensitivity of the solution would maximize the similarity between the true labels $\mathbf{y}$ and the inferred responses $\mathbf{y}^{\prime}$.

The sensitivity of the regression solution in the Hilbert space using a Tikhonov regularization in a general setting is studied in [57] and summarized in Theorem 1 and Corollary 1.
Theorem 1: Let $\kappa(.,$.$) be a kernel function and let the$ kernel matrix $\mathbf{K}$ corresponding to a set of observations $X$ be positive definite. Then, the optimal value of parameter $\delta>0$ with respect to the sensitivity of the regularized solution of the problem $E_{Z}+\delta\|\cdot\|_{K}^{2}$ is

$$
\delta_{o p t}=\frac{\lambda_{\min }\left(c(\mathbf{K})-\frac{c(\mathbf{K})+1}{2 \sqrt{c(\mathbf{K})}}\right)}{\frac{c(\mathbf{K})+1}{2 \sqrt{c(\mathbf{K})}}-1}
$$

where $E_{Z}=1 / n\|\mathbf{K} \boldsymbol{\alpha}-\mathbf{y}\|^{2}$

$\lambda_{\text {min }}$ denotes the smallest eigenvalue of the kernel matrix $\mathbf{K}$, and $c($.$) stands for the condition number of a matrix, defined$ as the ratio of the largest eigenvalue to the smallest one.

Corollary 1: In addition to the requirements of Theorem 1, if the kernel matrix is normalized, then the optimal value of parameter $\delta>0$ with respect to the sensitivity of the regularized solution of the problem $E_{Z}+\delta\|.\|_{K}^{2}$ is given as

$$
\delta_{\mathrm{opt}}=\frac{1}{1+\lambda_{\min }}-\frac{\lambda_{\min }\left(2-\sqrt{\lambda_{\min }}\right)}{2} .
$$

For a proof of the theorem and the corresponding corollary, [57].

As the kernel function used in this work is that of a radial basis function, the kernel matrix would be positive definite. Consequently, for a minimum sensitivity solution in the proposed approach, one may follow Algorithm 2, setting the regularization parameter based on (18) for a normalized kernel matrix.

\section{B. Sparse Regularization}

In addition to the widely used Tikhonov regularization, other regularization approaches encouraging sparseness of the solution have a rich history as a guideline for inference. The underlying motivation for seeking a sparse characterization is the desire to provide the simplest possible explanation of observation as a linear combination of as few as possible atoms from a given dictionary. One of the most celebrated instantiations of sparseness, the principle of minimum description length in model selection, requires that within a pool of models, the model that yields the most compact representation should be preferred for decision making. Such sparse methods select a small subset of relevant atoms to characterize the solution.

A sensible sparsity constraint to impose on the solution of the kernel spectral regression approach is the $l_{0}$-norm, defined as the number of nonzero elements in the solution. However, solving an $l_{0}$-regularized learning problem has been demonstrated to be NP-hard. The $l_{1}$-norm is shown to induce sparsity and can be used to approximate the optimal $l_{0}$-norm via convex relaxation. A least squares problem in the presence of an $l_{1}$ regularization term is known as Lasso in statistics and a basis pursuit in signal processing. Sparse $l_{1}$-norm models allow for scalable algorithms that can handle problems with a large number of parameters. Encouraging the solution of the kernel null-space approach to be sparse can conveniently be performed by enforcing an $l_{1}$-regularizer, i.e., $\mathcal{R}=\delta \sum_{i=1}^{n}\left|\alpha_{i}\right|$. Consequently, the objective function 


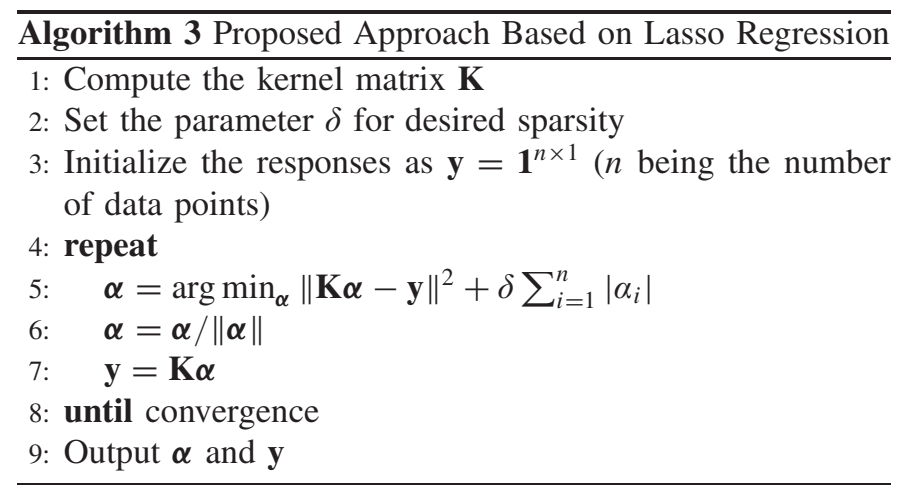

$P(\boldsymbol{\alpha}, \mathbf{y})$ in this case would be

$$
P(\boldsymbol{\alpha}, \mathbf{y})=\|\mathbf{K} \boldsymbol{\alpha}-\mathbf{y}\|^{2}+\delta \sum_{i=1}^{n}\left|\alpha_{i}\right| .
$$

The degree of the sparseness of a solution is controlled via parameter $\delta$. In a sparse formulation of the regression problem, each response $y_{i}$ is generated using only a few observations from the training set. In particular, if the solution $\alpha$ is very sparse, a large number of observations would have no contribution to the final solution, the immediate implication of which is a reduction in the computational complexity of the algorithm in the test phase. A further and more important consequence of forming a sparse model, as also suggested by other studies [58], is that of classification performance where a more compact model could improve performance compared with its nonsparse counterpart, especially in the presence of corrupted data.

Solving lasso problems efficiently is a subject of ongoing and fast-developing research. While the efficiency of solving the lasso problem is desirable, as this stage of the method is presumed to be performed offline, it has no impact on the efficiency of the proposed approach in the deployment phase. The complexity of the method in the test phase is governed by the degree of sparsity of the solution, i.e., $\boldsymbol{\alpha}$, and not by the procedure deriving such a solution. In this work, the least angle regression (LARS) algorithm [59] is used to find the optimal solution corresponding to $\boldsymbol{\alpha}=\arg \min _{\alpha}\|\mathbf{K} \boldsymbol{\alpha}-\mathbf{y}\|^{2}+$ $\delta \sum_{i=1}^{n}\left|\alpha_{i}\right|$. Using the LARS algorithm, solutions with all possible cardinalities on $\boldsymbol{\alpha}$ can be computed. For the lasso formulation, the proposed approach is given in Algorithm 3.

\section{Known Fraction of Contaminations}

In the discussions so far, it is assumed that the fraction of the data contaminated in the training set is not known in advance. In case the number of contaminations in the training set (denoted as $n_{0}$ ) is known, this information can be incorporated into the learning algorithm. This is realized by ranking training observations according to their compatibility with the model and identifying $n_{0}$ least compatible observations as nontarget samples, which results in the splitting of the training set into a target set and a nontarget set. For this purpose, at each iteration, the responses for all observations are sorted, and the smallest $n_{0}$ elements of $\mathbf{y}$ are set to zero, while others are set to one. This procedure essentially corresponds to updating

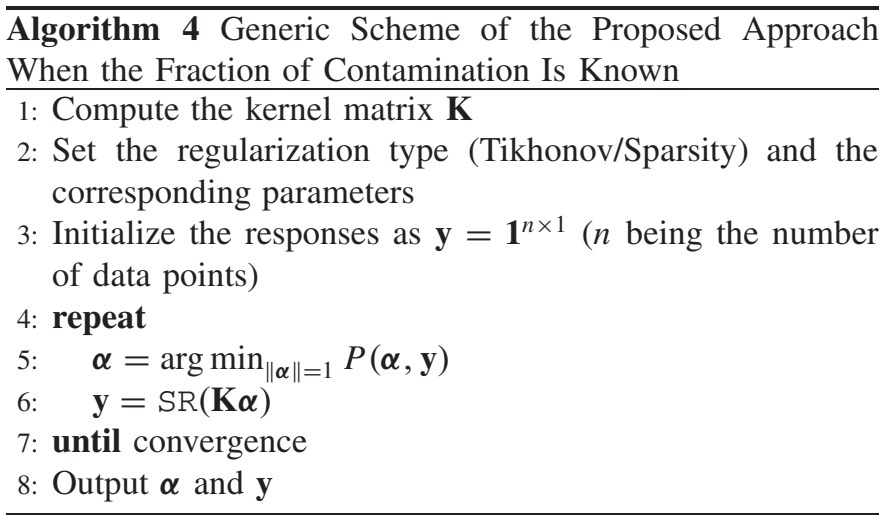

the initial normality assumption regarding observations, which is expected to improve the performance. In this respect, the samples corresponding to the $n_{0}$ samples that are less likely to be positive observations would form a nontarget set, which is then used for the refinement of the solution. The generic learning scheme, thus, obtained is given as Algorithm 4, where the $\operatorname{SR}($.$) routine corresponds to sorting and updating of the$ elements of an argument vector as described earlier. The minimization step $\boldsymbol{\alpha}=\arg \min _{\|\boldsymbol{\alpha}\|=1} P(\boldsymbol{\alpha}, \mathbf{y})$ in Algorithm 4 is performed as discussed earlier, depending on the specific type of regularization employed.

\section{Decision Strategy}

Once the optimal projection parameter $\boldsymbol{\alpha}$ is inferred, the projection of a test sample onto the feature subspace is realized as per (6). The projections of target samples in the proposed formulation are expected to lie at points closer to point 1 in the feature subspace, while those corresponding to nontarget samples are expected to lie at points closer to the origin. Consequently, the decision rule for a test sample $z$ is defined as

$$
\begin{aligned}
& f(z)=\sum_{i=1}^{n} \alpha_{i} \kappa\left(z, x_{i}\right) \geq \tau, \quad z \text { is } a \text { target object } \\
& f(z)=\sum_{i=1}^{n} \alpha_{i} \kappa\left(z, x_{i}\right)<\tau, \quad z \text { is an outlier }
\end{aligned}
$$

where $\tau$ is a threshold for deciding normality.

\section{EXPERIMENTAL EVALUATION}

In this section, an experimental evaluation of the proposed approach along with a comparison to the state-of-the-art methods is presented. On each data set, the training data include both positive and negative instances to simulate a contaminated training set.

\section{A. Data Sets}

1) Face: This data set is created to perform a toy experiment in face recognition. The data set contains face images of different individuals, and the task is to recognize a subject among others. For each subject, a one-class classifier is built using the training data associated with the subject under consideration, while all other subjects are assumed as outliers 
with respect to the built model. The experiment is repeated, in turn, for each subject in the data set. The features used for face image representation are obtained via the frontal-pose PAM deep CNN model [60] applied on the face bounding boxes. The data set is created out of the real-access videos of the Replay-Mobile data set [61] that provides face bounding boxes. In this work, ten subjects are used to form the data set where each subject is represented using 30 positive training instances. The number of negative training observations for each subject, i.e., contaminations, is also 30 images selected from all subjects other than the subject under consideration. The number of positive and negative test samples is similarly set to 30 images each.

2) MNIST: MNIST is a collection of $28 \times 28$ pixel images of handwritten digits 0-9 [62]. In our experiments, a single digit is considered as the target digit, while all others correspond to nontarget observations. In the experiments on this data set, the number of positive and negative training instances is set to 50 images each. Similarly, 50 positive and 50 negative images are included in the test set. The target class is assumed to be digit "3," while all other digits are selected as anomalies with respect to the target class.

3) Coil-100: The Coil-100 data set [63] contains 7200 images of 100 different objects. Each object has 72 images taken at pose intervals of five degrees, with the images being of size $32 \times 32$ pixels. In the experiments conducted on this data set, a single object is randomly chosen to be the target class, while all others are used as novelties. Raw pixel intensities are used as feature representations on this data set. The number of positive train and test instances for the target class is 36 each. Similarly, 36 negative train and 36 negative test observations are included in the experiments on this data set.

\section{B. Convergence Behavior}

In this section, the convergence behavior of the proposed block coordinate descent optimization method is analyzed. For this purpose, the proposed models are trained on the face, MNIST, and Coil-100 data sets. The error, defined as the norm of the deviation in $\alpha$ between two consecutive iterations, versus the number of iterations of the corresponding algorithms is plotted. The results are averaged over ten random splits of the data into training and test sets. A zero norm deviation would be indicative of the convergence of the optimization algorithm. The mean and std. (shaded regions in the figure) of the error versus the number of iterations are given in Figs. 1-3 for the face, MNIST, and Coil-100 data sets, respectively. As can be observed from Figs. 1-3, on all three data sets, the algorithm, on average, converges faster with the Tikhonov regularization compared with the sparse model. Nevertheless, regardless of the regularization type imposed on the solution, convergence is quite fast. The methods converge in just a few iterations (on average, in as few as five iterations).

\section{Effect of Regularization Parameter}

In this section, the effect of regularization parameters on the performance of the proposed methodology is evaluated.
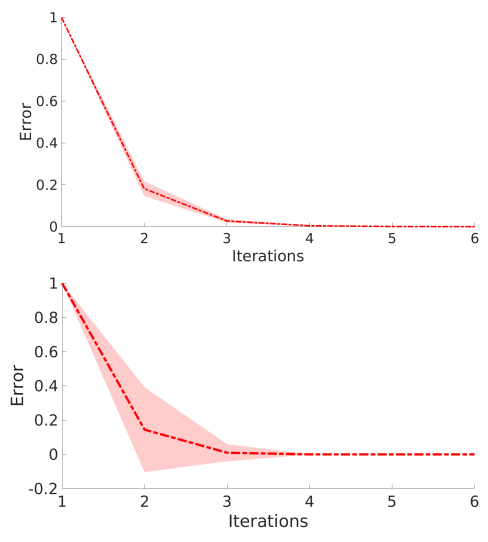

Fig. 1. Convergence behavior (mean \pm std) of the proposed iterative methods on the face data set. Top: Tikhonov regularization. Bottom: sparse regularization.
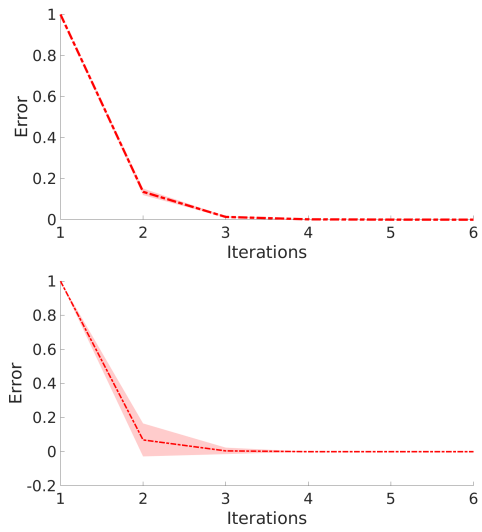

Fig. 2. Convergence behavior (mean \pm std) of the proposed iterative methods on the MNIST data set. Top: Tikhonov regularization. Bottom: sparse regularization.
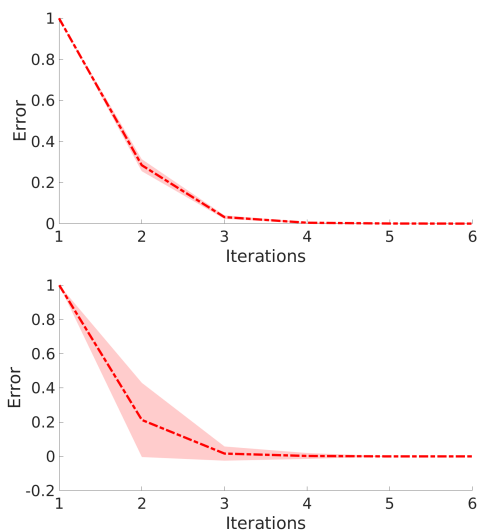

Fig. 3. Convergence behavior (mean \pm std) of the proposed iterative methods on the Coil-100 data set. Top: Tikhonov regularization. Bottom: sparse regularization.

The contamination is increased from $5 \%$ to $50 \%$ in steps of $5 \%$. Regarding the ridge regression formulation, the optimal Tikhonov regularization parameter along with three other parameters and the case where no regularization is imposed are considered. For the sparse model, the degree of sparseness is varied from $50 \%$ to $90 \%$ in steps of $10 \%$, where $n \%$ sparsity corresponds to the case where $n \%$ of variables in $\boldsymbol{\alpha}$ are zero. The results corresponding to this experiment for the 

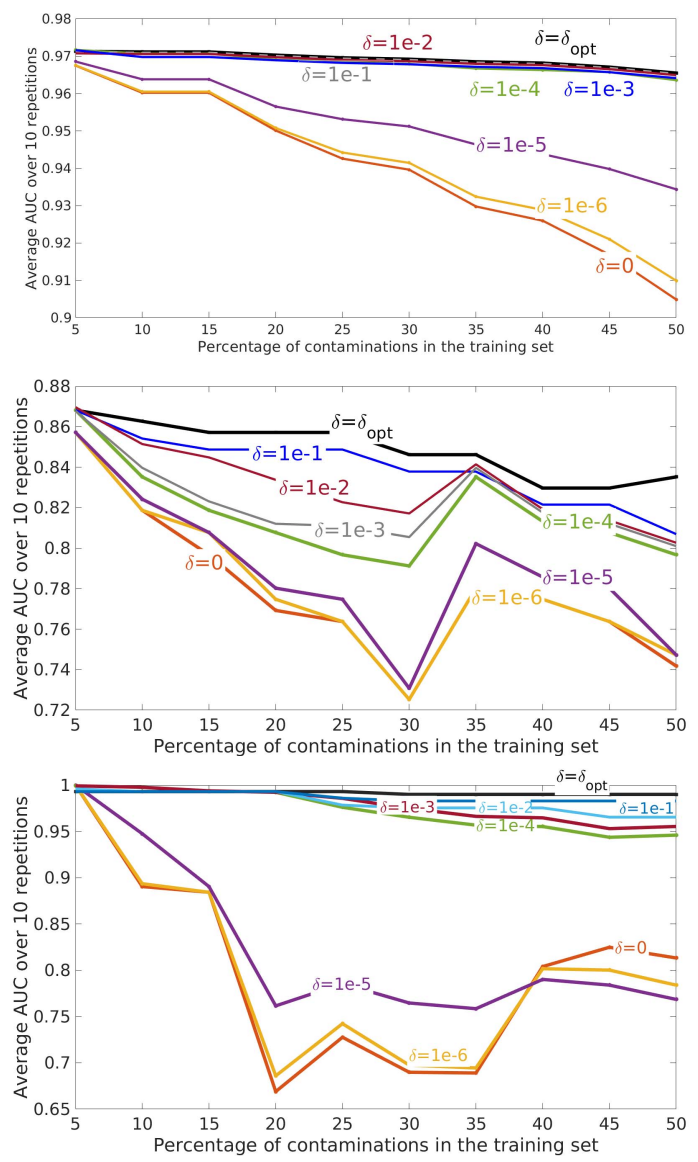

Fig. 4. Effect of varying the Tikhonov regularization on the performance of the proposed approach. From top to bottom: the face data set, the MNIST data set, and the Coil-100 data set.

Tikhonov regularized formulation and the sparse model on the three data sets are shown in Figs. 4 and 5, respectively. The plots present the average AUCs (the area under the receiver operating characteristic (ROC) curve) over ten random splits of data into the training and test sets. The ROC curve is created by plotting the true positive rate against the false-positive rate at various decision thresholds, and AUC represents a degree of separability by measuring the area under the ROC curve. The higher the AUC, the better the performance.

Regrading the Tikhonov regularized model, the worst performance corresponds to the case where no regularization is applied. Increasing the regularization parameter from zero toward the optimal value gradually enhances system robustness on all three data sets. Regarding the proposed sparse model, the sparser the solution toward the $90 \%$ sparsity level, the better the robustness of the method. This behavior is confirmed on all three data sets where a maximum sparseness of $90 \%$ achieves the best average overall performance over the entire range of contaminations. A common behavior for both types of regularization is that increasing the regularization effect improves robustness against contaminations toward higher levels of corruption at the cost of a small decrease in the performance for the lower percentages of contaminations. Nevertheless, the best average performance over the entire range of contaminations is obtained for the Tikhonov regularized
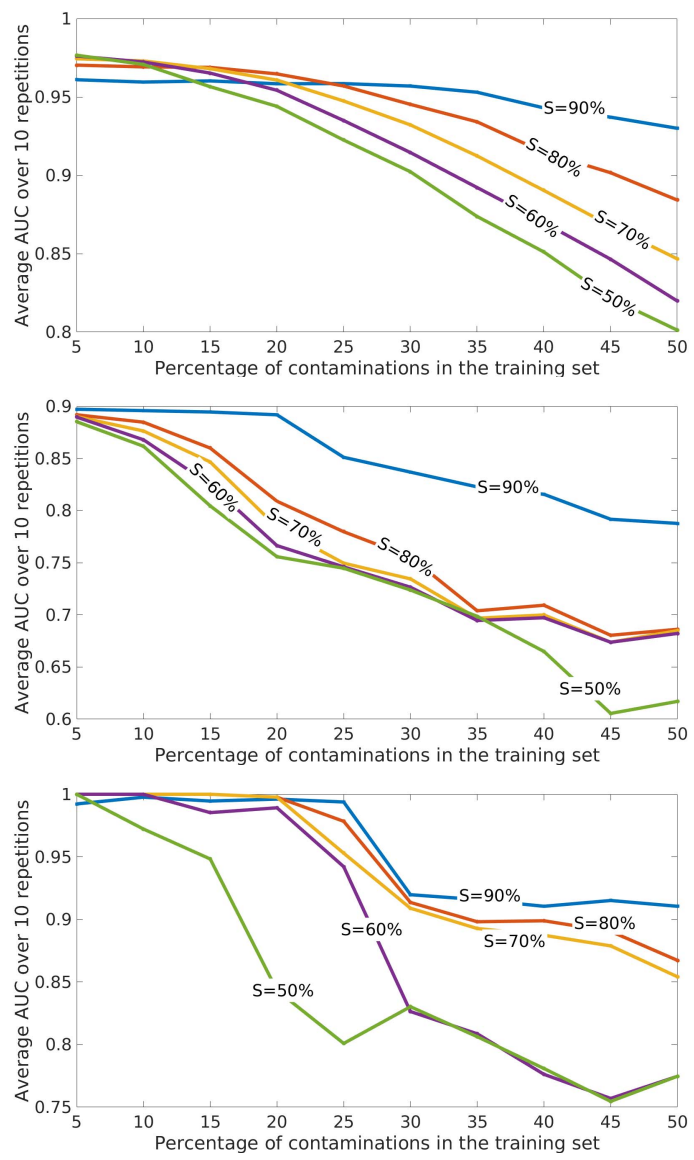

Fig. 5. Effect of varying sparseness on the performance of the proposed approach. From top to bottom: the face data set, the MNIST data set, and the Coil-100 data set.

model with the optimal regularization parameter and the sparse model set to a $90 \%$ sparsity level, indicating the effectiveness of regularization in improving the robustness of the kernel null-space approach.

\section{One-Class Classification in the Presence of Contaminations in the Training Set}

In this experiment, the proposed regularized null-space kernel spectral regression methods are evaluated on an OCC problem where the model is trained on a set of observations whose contamination is varied from $10 \%$ to $50 \%$ and then evaluated on a separate set of test samples. Such an evaluation scheme is commonly referred to as semisupervised OCC [2], where it is assumed that the training data incorporates labeled instances only for the normal class. In this experiment, the proposed methodology is compared against several other methods, including the following.

1) Tikh is the proposed robust spectral regression approach using a Tikhonov regularization term.

2) Spar is the proposed robust spectral regression approach using a sparsity inducing regularization term.

3) Org corresponds to the one-class kernel null FoleySammon transform. The methods presented in [28], [32], and [33] theoretically obtain the same result and only differ in terms of computational complexity. As the 
proposed approach is based on a kernel null-space formulation, the methods in [28], [32], and [33] serve as a baseline to gauge improvements obtained using the proposed approach.

4) SVDD is the support vector data description approach to solve the one-class classification problem [21]. As a widely used method, it serves as a second baseline for comparison.

5) GP is derived based on the Gaussian process regression and approximate Gaussian process classification [64], where, in this work, the predictive mean is used as the one class score.

6) $\mathrm{K}-\mathrm{m}$ is the $\mathrm{k}$-means clustering-based approach where $\mathrm{k}$ centers are assumed for the target observation. The novelty score of a sample is defined as the minimum distance of a query to cluster centers. The optimum value for parameter $k$ is experimentally set to 5 to achieve the best average performance over all data sets.

7) FB corresponds to the feature bagging algorithm [65], which detects outliers by bagging anomaly scores where the scores are generated by different individual outlier detection models operating on random subsets of input features. For each outlier detection model, the abnormal score is derived using a small subset from the set of available features. The base outlier detection method is that of the local outlier factor [66].

8) Pa. (Parzen) corresponds to the nonparametric density estimation approach based on Parzen-window estimators with Gaussian kernels [39] and works by sliding a window centered at each sample and computing a probability density function for each label. Samples for which the maximum probability is less than a threshold are identified as outliers.

1) Implementation Details: For the Org method, the implementation corresponding to the work in [32] is employed as it is the most efficient among other variants of the kernel nullspace technique. The SVDD method is based on the implementation provided in the data description toolbox [67]. The GP method is based on the implementation available publicly. ${ }^{1}$ Regarding the FB and Parzen approaches, the implementations provided in a supplement website ${ }^{2}$ are utilized. The $\mathrm{K}$-means clustering approach is based on its implementation in MATLAB 2017b. No preprocessing is applied on the features other than normalizing them to have a unit $l_{2}$-norm.

In the experiments on each data set, the data are randomly divided into the train and test sets, and the percentage of contaminations is increased from $10 \%$ to $50 \%$ of the total training data composed of positive and negative samples. Note that a $50 \%$ contamination in the training set represents a quite high degree of data corruption. The performance is then reported as the average AUCs over ten repetitions of the random splitting of the data into the train and test sets.

2) Results on the Face Data Set: The results in terms of average AUCs over ten random splittings of the data into the train and test sets on the face data set are plotted in Fig. 6 over

\footnotetext{
${ }^{1}$ https://github.com/erodner/gpocc

${ }^{2}$ https://github.com/gokererdogan/OutlierDetectionToolbox
}

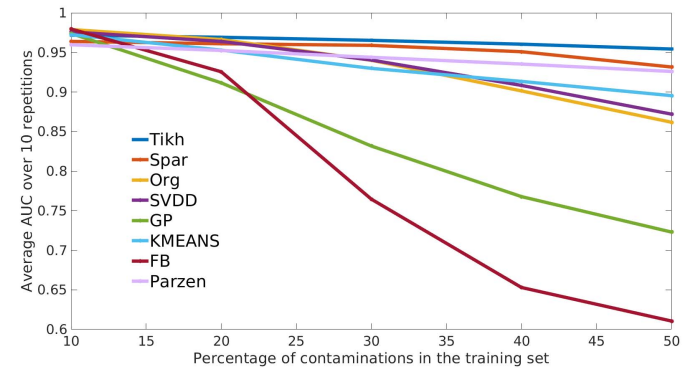

Fig. 6. Average AUCs for different methods over all subjects on the face data set.

TABLE I

Average Performance [IN TERms of AUC (\%)] OF DifFerent METHOdS IN AN OCC SCENARIO ON THE FACE DATA SET OVER THE FULl RANGE OF CONTAMINATION PERCENTAGES $(10 \%-50 \%)$

\begin{tabular}{lcccccccc}
\hline Method & Tikh & Spar & Org & SVDD & GP & K-means & FB & Parzen \\
\hline Subject \#1 & 96.20 & 94.71 & 90.09 & 90.74 & 80.23 & 87.99 & 78.58 & 90.01 \\
Subject \#2 & 97.03 & 95.86 & 93.61 & 92.31 & 84.66 & 95.58 & 84.18 & 95.32 \\
Subject \#3 & 99.95 & 99.30 & 97.80 & 97.88 & 87.77 & 99.25 & 83.86 & 99.63 \\
Subject \#4 & 99.82 & 99.51 & 98.59 & 98.72 & 89.80 & 96.83 & 80.51 & 99.66 \\
Subject \#5 & 95.84 & 93.21 & 93.28 & 91.47 & 81.23 & 90.80 & 78.78 & 92.67 \\
Subject \#6 & 95.51 & 95.20 & 94.24 & 93.93 & 83.35 & 89.79 & 73.90 & 94.39 \\
Subject \#7 & 98.04 & 97.24 & 95.83 & 96.63 & 87.55 & 95.13 & 85.12 & 95.48 \\
Subject \#8 & 99.88 & 97.41 & 89.43 & 93.95 & 84.55 & 99.67 & 82.10 & 99.74 \\
Subject \#9 & 99.77 & 99.13 & 96.60 & 97.89 & 89.92 & 98.86 & 82.96 & 99.60 \\
Subject \#10 & 95.68 & 94.68 & 94.55 & 92.86 & 85.76 & 94.80 & 77.82 & 95.00 \\
All Subjects & 96.80 & 95.89 & 93.61 & 93.65 & 84.80 & 94.30 & 78.50 & 94.82 \\
\hline
\end{tabular}

all subjects. The average AUCs (in percentage) for different subjects over the whole range of contaminations (from 10\% to 50\%) are reported in Table I. The corresponding AUC plots for each individual subject are provided as the Supplementary Material.

From Fig. 6 and Table I, it can be observed that the best performing method, on average, over the full range of contaminations is the proposed approach based on the Tikhonov regularization. Moreover, in terms of the average performance over all subjects, the second-best performing method is that of the proposed sparse approach. The improvement obtained using the proposed approach over the baseline method [28], [32], [33] over the entire range of contamination is more than $3 \%$ on average using a Tikhonov regularization and over $2 \%$ on average using a sparse regularization. However, as the percentage of contaminations in the training set increases, the effectiveness of the proposed regularization-based approach becomes more evident reaching more than $10 \%$ of improvement in terms of AUC for a $50 \%$ contamination in the training set.

3) Results on the MNIST Data Set: The results corresponding to different methods on the MNIST data set are provided in Fig. 7. From Fig. 7, it can be observed that the most robust method against contaminations in the training set is the proposed approach based on the Tikhonov regularization. The superiority of this formulation over other alternatives becomes more evident as more contaminations are included in the training set. The second-best performing method, on average, similar to the experiments on the face data set is again the proposed approach using a sparse regularization term.

4) Results on the Coil-100 Data Set: The results corresponding to different methods on the Coil-100 data set are presented in Fig. 8 in terms of average AUCs. From Fig. 8, 


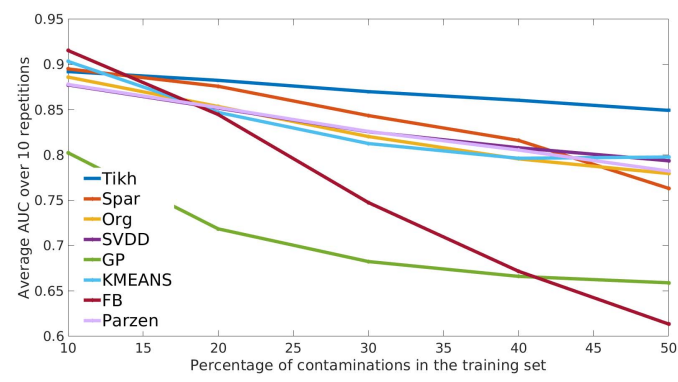

Fig. 7. Average AUCs for different methods on the MNIST data set.

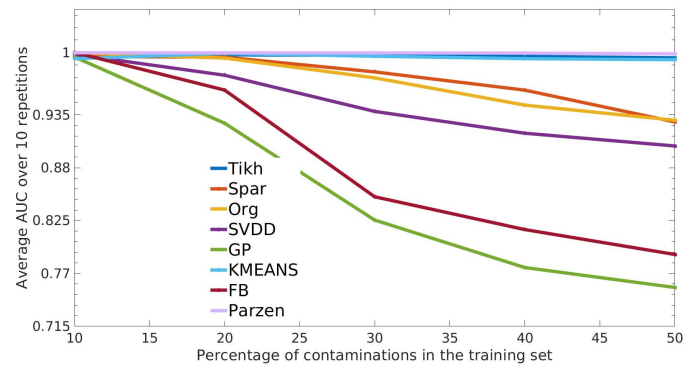

Fig. 8. Average AUCs for different methods on the Coil-100 data set.

TABLE II

Summary of the Average Performance [In Terms of AUC (\%)] OF DIFFERENT METHODS IN AN OCC SCENARIO ON THE MNIST AND COIL-100 Data SETS Over the Full Range of CONTAMINATION PERCENTAGES (10\%-50\%)

\begin{tabular}{lcccccccc}
\hline Method & Tikh & Spar & Org & SVDD & GP & K-m & FB & Pa. \\
\hline MNIST & 87.10 & 83.89 & 82.72 & 83.14 & 70.58 & 83.16 & 75.87 & 82.90 \\
Coil-100 & 99.64 & 97.18 & 96.83 & 94.63 & 85.57 & 99.48 & 88.32 & 99.93 \\
\hline
\end{tabular}

it can be observed that, interestingly, the two best performing methods are those of Parzen window and the proposed approach based on the Tikhonov regularization. Nevertheless, the proposed sparse model is not much worse. The results corresponding to the MNIST and Coil-100 data set are also summarized in Table II. As can be observed from Table II, the two best performing methods on the MNIST data set are the proposed methods based on the Tikhonov and Sparse regularization. In particular, the proposed approach based on the Tikhonov regularization, on average, is nearly $5 \%$ better than the original kernel null-space method [28], [32], [33]. On the Coil-100 data set, interestingly, the Parzen window method achieves the best average performance followed by the Tikhonov regularization-based approach.

\section{E. One-Class Classification in the Presence of Contaminations in the Training Set}

1) Known Fraction of Outliers: In the previous experiments, it was assumed that the fraction of contaminations in the training set is not known. In case such information is available, it can be utilized in the context of the proposed approaches. This is realized by detecting contaminations in the training set and forming a second class composed of the detected counterexamples that are used to refine the decision boundary.

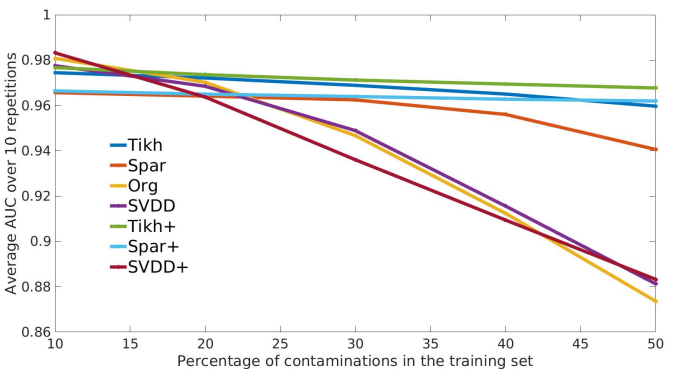

Fig. 9. Average AUC on the face data set over all subject-known fractions of contaminations in the training set.

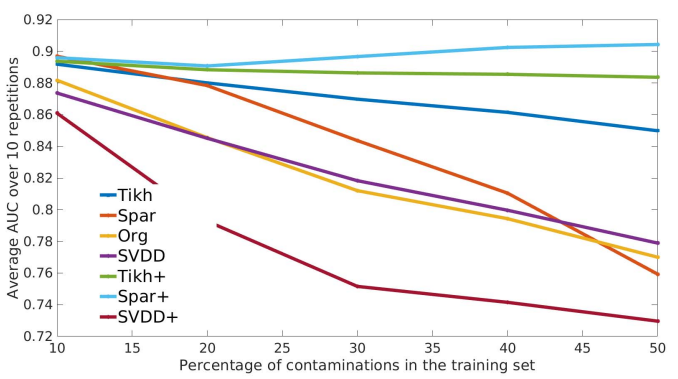

Fig. 10. Average AUC on the MNIST data set-known fraction of contaminations in the training set.

TABLE III

Summary of the Average Performance [in Terms of AUC (\%)] OF DIFFERENT METHODS IN AN OCC SCENARIO ON THE FACE, MNIST, AND COIL-100 Data SETS OVER THE Full RANGE of Contamination Percentages $(10 \%-50 \%)$ When THE FRACTION OF CONTAMINATIONS IS KNOWN

\begin{tabular}{lccccccc}
\hline Method & Tikh & Spar & Tikh+ & Spar+ & Org & SVDD & SVDD+ \\
\hline face & 96.80 & 95.78 & 97.17 & 96.40 & 93.67 & 93.84 & 93.51 \\
MNIST & 87.06 & 83.77 & 88.75 & 89.80 & 82.07 & 82.31 & 77.53 \\
Coil-100 & 99.34 & 97.37 & 99.52 & 99.33 & 97.34 & 94.93 & 97.52 \\
\hline
\end{tabular}

In this experiment, the number of outliers in the training set is provided as an additional input parameter, and the results are compared to the proposed naïve methods where no such information is available. Among other techniques, only the SVDD method is able to use such information for one-class learning. As a result, the methods included in this experiment are as follows.

1) Tikh (note that $n_{0}$ is not used).

2) Spar (again, $n_{0}$ is not used).

3) Tikh + is the proposed robust spectral regression approach based on the Tikhonov regularization exploiting the knowledge of $n_{0}$.

4) Spar+ is the proposed robust sparse spectral regression approach also exploiting the knowledge of $n_{0}$.

5) Org corresponds to the one-class kernel null FoleySammon transform [28], [32], [33].

6) SVDD ( $n_{0}$ is not used).

7) SVDD+ is the support vector data description approach making use of $n_{0}$.

The results corresponding to this experiment are presented in Figs. 9-11 for the face, MNIST, and Coil-100 data sets and also summarized in Table III.

From Figs. 9-11 and Table III, a number of conclusions can be drawn. First, it can be observed that the proposed methods 


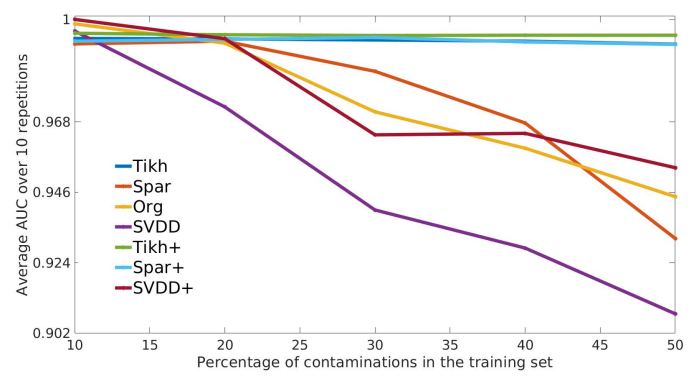

Fig. 11. Average AUC on the Coil-100 data set-known fraction of contaminations in the training set.

can effectively utilize the knowledge of $n_{0}$. This is evident by the fact that Tikh+ and Spar+ perform better that their naïve versions Tikh and Spar on all the three data sets examined. Second, the improvements obtained by Tikh+ and Spar+ are monotonically increasing with the increasing number of nontarget observations in the training set. This shows that in the proposed methodology, nontarget samples can play an effective role. Third, it can also be observed that, interestingly, on the MNIST data set, the additional information in terms of number of negative samples in the training set may even boost the performance of the Spar+ method. This is reflected in the average AUC of Spar+ method corresponding to a $50 \%$ corruption being higher than that of a $10 \%$ corruption in the data set.

2) Unknown Fraction of Outliers: In this section, we examine the possibility of automatically estimating the fraction of contamination from the data. The approach is based on the expectation that if the training data are contaminated, but we assume no contamination, a good fit would not be obtained. Similarly, if one assumes too much contamination, normal data will start mixing with negative samples resulting in a suboptimal fit. In this respect and in order to estimate the contamination percentage, we optimize the objective function $P$ for different hypothetical values of contamination $N_{0}$ and measure the normalized error as $\|\mathbf{K} \boldsymbol{\alpha}-\mathbf{y}\|^{2} / N_{o}$, where $\mathbf{y}$ is also normalized to have a unit norm. The value of $N_{o}$ that gives the minimum error is then considered as an estimate of the true $n_{0}$.

Given an estimate of $n_{0}$, the rest of the procedure is similar to that of Section V-E1. Applying the abovementioned idea to the Tikhonov-regularized approach (denoted as A-Tikh), a similar set of experiments to that of Section V-E1 is performed, including the A-Tikh approach. The results of the comparison of different variants of the Tikhonov and sparse regularized methods on the face data set are presented in Fig. 12. One may observe that the A-Tikh approach performs better than Tikh and Spar, where no automatic mechanism for detecting outliers is available. Moreover, the A-Tikh performs better than the Spar+ method that uses the knowledge of $n_{0}$. The effectiveness of the A-Tikh method is accentuated by its almost similar performance to Tikh + for which the percentage of contaminations is provided as an input parameter. For space constraints, the results are shown only for the face data. However, the same behavior was observed on the other data sets too.

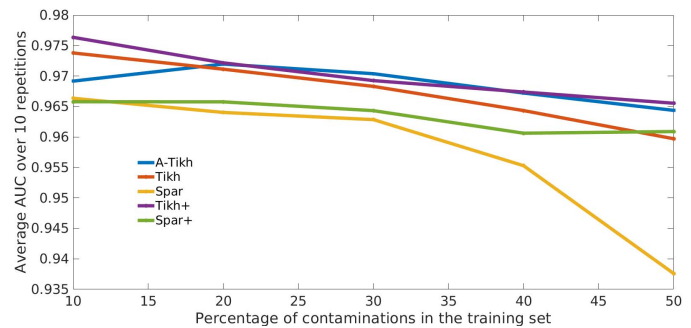

Fig. 12. Average AUC on the face data set over all subjects.

\section{F. Unsupervised Observation Ranking}

In a final set of experiments, the proposed regularized kernel spectral regression methods are evaluated in an observation ranking paradigm. As noted earlier, the original null-space kernel one-class methods are unable to provide a ranking of training observations. Nevertheless, this limitation is removed in the proposed regularized methods by imposing an additional regularization term while, at the same time, updating observation label confidences via an iterative alternating minimization technique. The methods included in the comparison in this set of experiments are the state-of-the-art methods for unsupervised observation ranking and outlier detection. Consistent with the literature [2], unsupervised methods refer to those approaches that do not require training data and make the implicit assumption that normal instances are more frequently present than anomalies in a data set. Consequently, the methods included in this experiment are those that are specifically designed to operate on a given set of contaminated samples and provide a compatibility ranking. As such, different methods in this experiment are utilized to rank observations in the training set only. The methods compared in this experiment are as follows.

1) Tikh.

2) Spar.

3) DPCP is a method for learning a linear subspace from data corrupted by outliers based on a nonconvex $l_{1}$ optimization [50]. It is shown that DPCP can tolerate as many outliers as the square of the number of inliers, thus improving upon other robust PCA methods.

4) $\mathrm{OP}$ is an efficient convex optimization-based algorithm [51] to perform a robust principal component analysis that recovers the exact optimal low-dimensional subspace under mild assumptions and identifies the corrupted points.

5) FMS is a nonconvex robust subspace recovery approach [52], designed to be least affected by corruptions in the training set, and has been demonstrated to converge to close vicinity of the correct subspace within few iterations.

6) SRO obtains a weighted directed graph, defines a Markov Chain via self-representation, and identifies outliers via random walks [53]. The SRO method can be considered as one of the leading unsupervised approaches for novelty detection.

The results, in terms of average AUC, corresponding to this experiment are provided in Figs. 13-15 for 


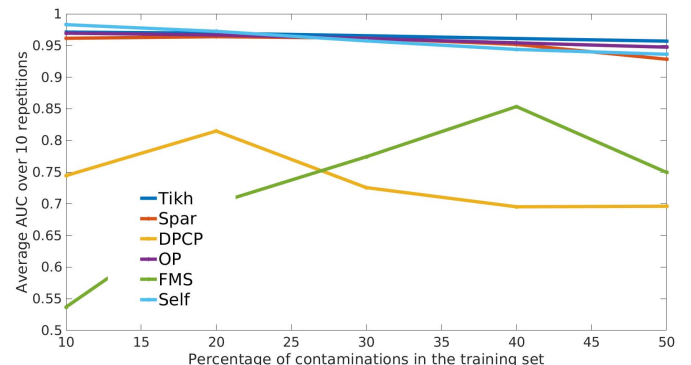

Fig. 13. Average AUC over all subjects on the face data set corresponding to a ranking of training samples.

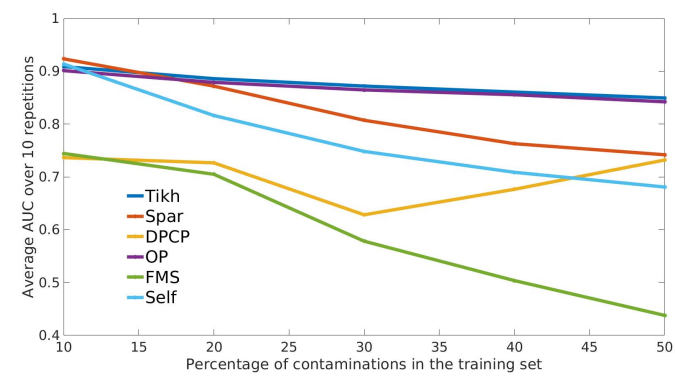

Fig. 14. Average AUC on the MNIST data set corresponding to a ranking of training samples.

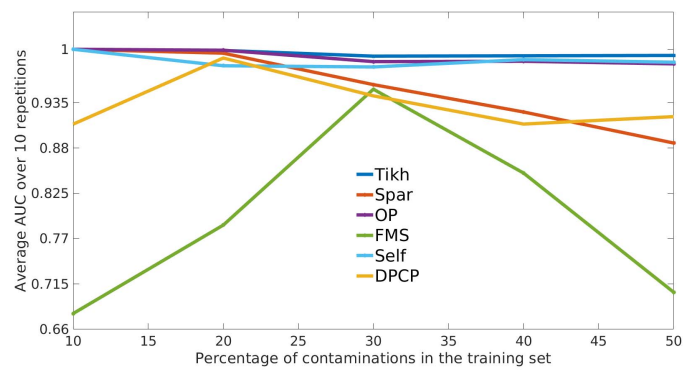

Fig. 15. Average AUC on the Coil-100 data set corresponding to a ranking of training samples.

the face, MNIST, and Coil-100 data sets, respectively, and also summarized in Table IV. From Table IV and Figs. 13-15, it can be observed that the Tikhonov regularization-based approach is the top performer among other competitors on all three data sets in an unsupervised observation ranking and novelty detection scenario. The proposed sparsity-based approach performs less well compared to the Tikhonov regressionbased formulation. Nevertheless, it still performs better than some other competitors, including the recently proposed DPCP and FMS approaches.

\section{G. Computational Complexity}

In Algorithm 2, in practice, computing the inverse matrix is performed outside the iteration loop as matrix $\mathbf{K}+\delta \mathbf{I}_{n}$ is independent of parameter $\boldsymbol{\alpha}$ and the responses $\mathbf{y}$. As such, the main loop of the Tikhonov regularized approach only incorporates matrix-vector multiplication and vector normalization. In Algorithm 3, the computational complexity of the
TABLE IV

Summary of the Average Performance [in Terms of AUC (\%)] of DIFFERENT METHODS IN UNSUPERVISED OBSERVATION RANKING ON THE FACE, MNIST, AND COIL-100 DATA SETS OVER THE FulL RANGE OF CONTAMINATION PERCENTAGES (10\%-50\%)

\begin{tabular}{lcccccc}
\hline Method & Tikh & Spar & DPCP & OP & FMS & SRO \\
\hline face & 96.48 & 95.34 & 73.52 & 95.98 & 72.30 & 95.85 \\
MNIST & 87.52 & 82.14 & 69.99 & 86.84 & 59.38 & 77.35 \\
Coil-100 & 99.50 & 95.25 & 93.39 & 99.03 & 79.44 & 98.61 \\
\hline
\end{tabular}

TABLE V

Average Running Times (train + test) Over Ten RePetitions (IN s) of Different Methods in OCC on the Face Data Set

\begin{tabular}{cccccccc}
\hline Method & Tikh & Spar & SVDD & GP & K-m & FB & Pa \\
\hline Runtime & 0.009 & 0.21 & 2.68 & 1.20 & 0.03 & 1.89 & 1.40 \\
\hline
\end{tabular}

TABLE VI

Average RunNing Times Over Ten Repetitions (IN s) OF Different METHODS IN UNSUPERVISED OBSERVATION RANKING on the Face Data Set

\begin{tabular}{ccccccc}
\hline Method & Tikh & Spar & DPCP & OP & FMS & SRO \\
\hline Runtime & 0.007 & 0.20 & 5.27 & 0.68 & 1.72 & 0.07
\end{tabular}

TABLE VII

Computational Complexities of DifFerent Methods Per Each ItERATION. (ABbreViations ARE DEFInED Within the MAin TEXT)

\begin{tabular}{cccccc}
\hline Tikh & Spar & K-m & FB & Pa & GP \\
\hline $\mathcal{O}\left(n^{2}\right)$ & $\mathcal{O}\left(n^{3}\right)$ & $\mathcal{O}(n)$ & $\mathcal{O}\left(n^{2}\right)$ & $\mathcal{O}\left(n^{2}\right)$ & $\mathcal{O}\left(n^{3}\right)$ \\
\hline \hline DPCP & OP & FMS & SRO & & \\
\hline $\mathcal{O}\left(n^{2}\right)$ & $\mathcal{O}(n D d)$ & $\mathcal{O}\left(n^{3}\right)$ & $\mathcal{O}\left(n^{3}\right)$ & & \\
\hline
\end{tabular}

LARS algorithm is $\mathcal{O}\left(n^{3}\right)$ for the training phase. However, the complexity of the method in the operational phase is governed by the degree of sparsity of the solution (i.e., $\boldsymbol{\alpha}$ ). The average running times of different algorithms in different scenarios are reported in Tables V and VI. Note that the original source codes provided for each method, as described previously, were used to measure the running times of different methods. The implementation of the proposed approaches is based on unoptimized MATLAB codes, running on a 64-bit 4.00-GHz Intel Core-i7 machine with 32-GB memory. The computational complexities of different methods per each iteration (in the case of an iterative method) are tabulated in Table VII, where $n$ is the number of training observations, while $D$ and $d$ correspond to the original dimensionality of features and the reduced dimensionality in the subspace. Please note that the number of iterations for different algorithms varies widely, directly impacting on the running times of the algorithms.

\section{CONCLUSION}

Two limitations of the null-space kernel Fisher analysis for OCC, namely, its sensitivity to training set contamination and its inability to rank training samples, are addressed. We propose regularized variants of a regression-based formulation 
of the learning problem (Tikhonov and sparsity) where both projection parameters and object labels are inferred iteratively. Through experiments on different data sets, it was demonstrated that: 1) the proposed regularization approach combined with the alternating optimization mechanism is effective in robustifying the baseline method; 2) the proposed iterative ridge regression-based formulation posing one-class learning as a sensitivity analysis problem is the top performer among other competitors; and 3) the performance of both alternative regularization schemes is boosted by automatically detecting negative samples in the training set and forming a subset of counterexamples for enhanced training.

The proposed methodology was evaluated in an OCC paradigm by assessing its generalization capability as well as in an observation ranking scheme to detect outliers in a given data set.

\section{REFERENCES}

[1] H. He and E. A. Garcia, "Learning from imbalanced data," IEEE Trans. Knowl. Data Eng., vol. 21, no. 9, pp. 1263-1284, Sep. 2009.

[2] V. Chandola, A. Banerjee, and V. Kumar, "Anomaly detection: A survey," ACM Comput. Surv., vol. 41, no. 3, pp. 15:1-15:58, Jul. 2009.

[3] P. Nader, P. Honeine, and P. Beauseroy, " $l_{p}$-norms in one-class classification for intrusion detection in SCADA systems," IEEE Trans. Ind. Informat., vol. 10, no. 4, pp. 2308-2317, Nov. 2014.

[4] A. Beghi, L. Cecchinato, C. Corazzol, M. Rampazzo, F. Simmini, and G. Susto, "A one-class SVM based tool for machine learning novelty detection in HVAC chiller systems," IFAC Proc. Volumes, vol. 47, no. 3, pp. 1953-1958, 2014. [Online]. Available: http://www.sciencedirect. com/science/article/pii/S1474667016418999

[5] S. Budalakoti, S. Budalakoti, A. N. Srivastava, M. E. Otey, and M. E. Otey, "Anomaly detection and diagnosis algorithms for discrete symbol sequences with applications to airline safety," IEEE Trans. Syst., Man, Cybern. C, Appl. Rev., vol. 39, no. 1, pp. 101-113, Jan. 2009.

[6] S. Kamaruddin and V. Ravi, "Credit card fraud detection using big data analytics: Use of PSOAANN based one-class classification," in Proc. Int. Conf. Informat. Anal. (ICIA), New York, NY, USA, 2016, pp. 33:1-33:8. [Online]. Available: http://doi.acm.org/10.1145/2980258.2980319

[7] G. G. Sundarkumar, V. Ravi, and V. Siddeshwar, "One-class support vector machine based undersampling: Application to churn prediction and insurance fraud detection," in Proc. IEEE Int. Conf. Comput. Intell. Comput. Res. (ICCIC), Dec. 2015, pp. 1-7.

[8] M. Yu, Y. Yu, A. Rhuma, S. M. R. Naqvi, L. Wang, and J. A. Chambers, "An online one class support vector machine-based person-specific fall detection system for monitoring an elderly individual in a room environment," IEEE J. Biomed. Health Informat., vol. 17, no. 6, pp. 1002-1014, Nov. 2013.

[9] A. Rabaoui, M. Davy, S. Rossignol, and N. Ellouze, "Using one-class SVMs and wavelets for audio surveillance," IEEE Trans. Inf. Forensics Security, vol. 3, no. 4, pp. 763-775, Dec. 2008.

[10] S. S. Khan and M. G. Madden, "One-class classification: Taxonomy of study and review of techniques," Knowl. Eng. Rev., vol. 29, no. 3, pp. 345-374, Jun. 2014.

[11] D. Tax, "One-class classification: Concept-learning in the absence of counter-examples," Ph.D. dissertation, Dept. Appl. Sci., Pattern Recognit. Group, Delft Univ. Technol., Delft, The Netherlands, 2001.

[12] M. A. F. Pimentel, D. A. Clifton, L. Clifton, and L. Tarassenko, "A review of novelty detection," Signal Process., vol. 99, pp. 215-249, Jun. 2014. [Online]. Available: http://www.sciencedirect.com/science/ Earticle/pii/S016516841300515X

[13] J. Kittler et al., "Domain anomaly detection in machine perception: A system architecture and taxonomy," IEEE Trans. Pattern Anal. Mach. Intell., vol. 36, no. 5, pp. 845-859, May 2014.

[14] D. M. J. Tax and R. P. W. Duin, "Combining one-class classifiers," in Proc. 2nd Int. Workshop Multiple Classifier Syst. (MCS), London, U.K.: Springer-Verlag, 2001, pp. 299-308.

[15] L. Friedland, A. Gentzel, and D. Jensen, "Classifier-adjusted density estimation for anomaly detection and one-class classification," in Proc. SIAM Int. Conf. Data Mining, Apr. 2014, pp. 578-586.
[16] H. Hoffmann, "Kernel PCA for novelty detection," Pattern Recognit., vol. 40, no. 3, pp. 863-874, Mar. 2007. [Online]. Available: http://www.sciencedirect.com/science/article/pii/S0031320306003414

[17] N. Japkowicz, "Concept learning in the absence of counterexamples: An autoassociation-based approach to classification," Ph.D. dissertation, Dept. Comput. Sci., Rutgers Univ., New Brunswick, NJ, USA, 1999.

[18] M. Sabokrou, M. Fathy, and M. Hoseini, "Video anomaly detection and localisation based on the sparsity and reconstruction error of auto-encoder," Electron. Lett., vol. 52, no. 13, pp. 1122-1124, Jun. 2016

[19] S. R. Arashloo, J. Kittler, and W. Christmas, "An anomaly detection approach to face spoofing detection: A new formulation and evaluation protocol," IEEE Access, vol. 5, pp. 13868-13882, 2017.

[20] B. Song, P. Li, J. Li, and A. Plaza, "One-class classification of remote sensing images using kernel sparse representation," IEEE J. Sel. Topics Appl. Earth Observ. Remote Sens., vol. 9, no. 4, pp. 1613-1623, Apr. 2016.

[21] D. M. J. Tax and R. P. W. Duin, "Support vector data description," Mach. Learn., vol. 54, no. 1, pp. 45-66, Jan. 2004, doi: 10.1023/B:MACH. 0000008084.60811.49.

[22] B. Schölkopf, J. C. Platt, J. Shawe-Taylor, A. J. Smola, and R. C. Williamson, "Estimating the support of a high-dimensional distribution," Neural Comput., vol. 13, no. 7, pp. 1443-1471, Jul. 2001, doi: 10.1162/089976601750264965.

[23] P. Casale, O. Pujol, and P. Radeva, "Approximate convex hulls family for one-class classification," in Multiple Classifier Systems, C. Sansone, J. Kittler, and F. Roli, Eds. Berlin, Germany: Springer, 2011, pp. $106-115$.

[24] D. Fernandez-Francos, O. Fontenla-Romero, and A. Alonso-Betanzos, "One-class convex hull-based algorithm for classification in distributed environments," IEEE Trans. Syst., Man, Cybern. Syst., vol. 50, no. 2, pp. 386-396, Feb. 2020.

[25] A. Ypma and R. P. W. Duin, "Support objects for domain approximation," in Proc. ICANN, L. Niklasson, M. Bodén, and T. Ziemke, Eds. London, U.K.: Springer, 1998, pp. 719-724.

[26] V. Roth, "Outlier detection with one-class kernel Fisher discriminants," in Advances in Neural Information Processing Systems, L. K. Saul, Y. Weiss, and L. Bottou, Eds. Cambridge, MA, USA: MIT Press, 2005, pp. 1169-1176. [Online]. Available: http://papers.nips.cc/paper/2656outlier-detection-with-one-class-kernel-fisher-discriminants.pdf

[27] V. Roth, "Kernel Fisher discriminants for outlier detection," Neural Comput., vol. 18, no. 4, pp. 942-960, Apr. 2006, doi: 10.1162/ 089976606775774679.

[28] P. Bodesheim, A. Freytag, E. Rodner, M. Kemmler, and J. Denzler, "Kernel null space methods for novelty detection," in Proc. IEEE Conf. Comput. Vis. Pattern Recognit., Jun. 2013, pp. 3374-3381.

[29] F. Dufrenois, "A one-class kernel Fisher criterion for outlier detection," IEEE Trans. Neural Netw. Learn. Syst., vol. 26, no. 5, pp. 982-994, May 2015.

[30] F. Dufrenois and J. C. Noyer, "Formulating robust linear regression estimation as a one-class LDA criterion: Discriminative hat matrix," IEEE Trans. Neural Netw. Learn. Syst., vol. 24, no. 2, pp. 262-273, Feb. 2013

[31] P. Juszczak, D. M. J. Tax, E. Pękalska, and R. P. W. Duin, "Minimum spanning tree based one-class classifier," Neurocomputing, vol. 72, nos. 7-9, pp. 1859-1869, Mar. 2009. [Online]. Available: http://www. sciencedirect.com/science/article/pii/S0925231208003238

[32] S. R. Arashloo and J. Kittler, "One-class kernel spectral regression," 2018, arXiv:1807.01085. [Online]. Available: http://arxiv. org/abs/1807.01085

[33] J. Liu, Z. Lian, Y. Wang, and J. Xiao, "Incremental kernel null space discriminant analysis for novelty detection," in Proc. IEEE Conf. Comput. Vis. Pattern Recognit. (CVPR), Jul. 2017, pp. 4123-4131.

[34] X. Huang, J. Xu, and G. Guo, "Incremental kernel null foley-sammon transform for person re-identification," in Proc. 24th Int. Conf. Pattern Recognit. (ICPR), Aug. 2018, pp. 1683-1688.

[35] V. Mygdalis, A. Iosifidis, A. Tefas, and I. Pitas, "Laplacian one class extreme learning machines for human action recognition," in Proc. IEEE 18th Int. Workshop Multimedia Signal Process. (MMSP), Sep. 2016, pp. $1-5$.

[36] L.-F. Chen, H.-Y.-M. Liao, M.-T. Ko, J.-C. Lin, and G.-J. Yu, "A new LDA-based face recognition system which can solve the small sample size problem," Pattern Recognit., vol. 33, no. 10, pp. 1713-1726, Oct. 2000. [Online]. Available: http://www.sciencedirect.com/science/ article/pii/S0031320399001399 
[37] D. Chu and G. S. Thye, "A new and fast implementation for null space based linear discriminant analysis," Pattern Recognit., vol. 43, no. 4, pp. 1373-1379, Apr. 2010. [Online]. Available: http://www. sciencedirect.com/science/article/pii/S003132030900377X

[38] L. Tarassenko, P. Hayton, N. Cerneaz, and M. Brady, "Novelty detection for the identification of masses in mammograms," in Proc. 4th Int. Conf. Artif. Neural Netw., Jun. 1995, pp. 442-447.

[39] D.-Y. Yeung and C. Chow, "Parzen-window network intrusion detectors," Object Recognit. Supported User Interact. Service Robots, vol. 4, Aug. 2002, pp. 385-388.

[40] V. Barnett and T. Lewis, Outliers in Statistical Data, 2nd ed. Hoboken, NJ, USA: Wiley, 1978

[41] C. Bishop, Neural Networks for Pattern Recognition. London, U.K.: Oxford Univ. Press, 1995.

[42] G. A. Carpenter, S. Grossberg, and D. Rosen, "ART 2-A: An adaptive resonance algorithm for rapid category learning and recognition," Boston Univ., Boston, MA, USA, Tech. Rep. CAS/CNS-TR-91011, 1991. [Online]. Available: https://open.bu.edu/handle/2144/2066

[43] N. Japkowicz, C. Myers, and M. Gluck, "A novelty detection approach to classification," in Proc. 14th Int. Joint Conf. Artif. Intell. (IJCAI), vol. 1. San Francisco, CA, USA: Morgan Kaufmann, 1995, pp. 518-523.

[44] S. Günter, N. N. Schraudolph, and S. V. N. Vishwanathan, "Fast iterative kernel principal component analysis," J. Mach. Learn. Res., vol. 8 , pp. 1893-1918, Aug. 2007.

[45] N. Kwak, "Principal component analysis based on L1-norm maximization," IEEE Trans. Pattern Anal. Mach. Intell., vol. 30, no. 9 , pp. 1672-1680, Sep. 2008.

[46] P. Bodesheim, A. Freytag, E. Rodner, and J. Denzler, "Local novelty detection in multi-class recognition problems," in Proc. IEEE Winter Conf. Appl. Comput. Vis., Jan. 2015, pp. 813-820.

[47] F. Dufrenois and J. C. Noyer, "One class proximal support vector machines," Pattern Recognit., vol. 52, pp. 96-112, Apr. 2016. [Online]. Available: http://www.sciencedirect.com/science/article/pii/ S0031320315003672

[48] F. Dufrenois and J. C. Noyer, "A null space based one class kernel Fisher discriminant," in Proc. Int. Joint Conf. Neural Netw. (IJCNN), Jul. 2016 pp. 3203-3210.

[49] D. Cai, X. He, and J. Han, "Speed up kernel discriminant analysis," VLDB J., vol. 20, no. 1, pp. 21-33, Feb. 2011.

[50] Z. Zhu, Y. Wang, D. Robinson, D. Naiman, R. Vidal, and M. Tsakiris, "Dual principal component pursuit: Improved analysis and efficient algorithms," in Advances in Neural Information Processing Systems, S. Bengio, H. Wallach, H. Larochelle, K. Grauman, N. Cesa-Bianchi, and R. Garnett, Eds. Red Hook, NY, USA: Curran Associates, 2018, pp. 2175-2185. [Online]. Available: http://papers.nips.cc/paper/ 7486-dual-principal-component-pursuit-improved-analysis-andefficient-algorithms.pdf

[51] H. Xu, C. Caramanis, and S. Sanghavi, "Robust PCA via outlier pursuit," IEEE Trans. Inf. Theory, vol. 58, no. 5, pp. 3047-3064, May 2012.

[52] G. Lerman and T. Maunu, "Fast, robust and non-convex subspace recovery," Inf. Inference: A J. IMA, vol. 7, no. 2, pp. 277-336, Jun. 2018, doi: 10.1093/imaiai/iax012.

[53] C. You, D. P. Robinson, and R. Vidal, "Provable self-representation based outlier detection in a union of subspaces," in Proc. IEEE Conf. Comput. Vis. Pattern Recognit. (CVPR), Jul. 2017, pp. 4323-4332.

[54] G. R. G. Lanckriet, N. Cristianini, P. Bartlett, L. El Ghaoui, and M. I. Jordan, "Learning the kernel matrix with semidefinite programming," J. Mach. Learn. Res., vol. 5, pp. 27-72, Jan. 2004.

[55] F. Yan, J. Kittler, K. Mikolajczyk, and A. Tahir, "Non-sparse multiple kernel Fisher discriminant analysis," J. Mach. Learn. Res., vol. 13, no. 1, pp. 607-642, Mar. 2012.

[56] G. W. Stewart, Matrix Algorithms: Basic Decompositions, vol. 1. Philadelphia, PA, USA: SIAM, 2001.

[57] K. Hlaváčková-Schindler, "Tikhonov regularization parameter in reproducing kernel Hilbert spaces with respect to the sensitivity of the solution," in Artificial Neural Networks, V. Kưrková, R. Neruda, and J. Koutník, Eds. Berlin, Germany: Springer, 2008, pp. 215-224.

[58] J. Wright, A. Y. Yang, A. Ganesh, S. S. Sastry, and Y. Ma, "Robust face recognition via sparse representation," IEEE Trans. Pattern Anal. Mach. Intell., vol. 31, no. 2, pp. 210-227, Feb. 2009.

[59] B. Efron, T. Hastie, I. Johnstone, and R. Tibshirani, "Least angle regression," Ann. Statist., vol. 32, no. 2, pp. 407-451, 2004.

[60] I. Masi et al., "Learning pose-aware models for pose-invariant face recognition in the wild," IEEE Trans. Pattern Anal. Mach. Intell., vol. 41, no. 2, pp. 379-393, Feb. 2019.
[61] A. Costa-Pazo, S. Bhattacharjee, E. Vazquez-Fernandez, and S. Marcel, "The replay-mobile face presentation-attack database," in Proc. Int Conf. Biometrics Special Interest Group (BIOSIG), Sep. 2016, pp. 1-7.

[62] Y. Lecun, L. Bottou, Y. Bengio, and P. Haffner, "Gradient-based learning applied to document recognition," Proc. IEEE, vol. 86, no. 11, pp. 2278-2324, Nov. 1998.

[63] S. A. Nene, S. K. Nayar, and H. Murase, "Columbia object image library (COIL-100)," Columbia Univ., New York, NY, USA, Tech. Rep. CUCS006-96, Feb. 1996.

[64] M. Kemmler, E. Rodner, E.-S. Wacker, and J. Denzler, "One-class classification with Gaussian processes," Pattern Recognit., vol. 46, no. 12 , pp. 3507-3518, Dec. 2013. [Online]. Available: http://www. sciencedirect.com/science/article/pii/S0031320313002574

[65] A. Lazarevic and V. Kumar, "Feature bagging for outlier detection," in Proc. 11th ACM SIGKDD Int. Conf. Knowl. Discovery Data Mining, Chicago, IL, USA, Aug. 2005, pp. 157-166.

[66] M. Breunig, H.-P. Kriegel, R. T. Ng, and J. Sander, "LOF: Identifying density-based local outliers," in Proc. ACM SIGMOD Int. Conf. Manage. Data, 2000, pp. 93-104.

[67] D. Tax, "Ddtools, the data description toolbox for MATLAB, version 2.1.3," Technical Univ. Delft, Delft, The Netherlands, Tech. Rep., Jan. 2018

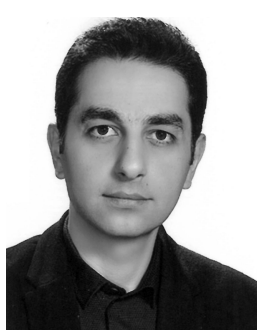

Shervin Rahimzadeh Arashloo received the $\mathrm{Ph} . \mathrm{D}$. degree from the Centre for Vision, Speech and Signal Processing, University of Surrey, Guildford, U.K., in 2010.

$\mathrm{He}$ is an Assistant Professor with the Department of Computer Engineering, Bilkent University, Ankara, Turkey, and a Visiting Research Fellow with the Centre for Vision, Speech and Signal Processing, University of Surrey. His research interests include secured biometrics, anomaly detection, and graphical models with applications to image and video analyses.

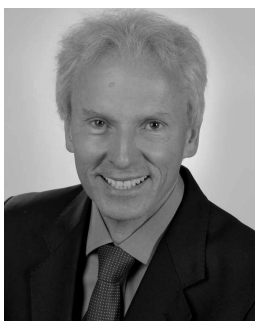

Josef Kittler (Life Member, IEEE) received the B.A., Ph.D., and D.Sc. degrees from the University of Cambridge, Cambridge, U.K., in 1971, 1974, and 1991, respectively.

$\mathrm{He}$ is currently a Professor of machine intelligence with the Centre for Vision, Speech and Signal Processing, Department of Electronic Engineering, University of Surrey, Guildford, U.K. He conducts research in biometrics, video and image database retrieval, medical image analysis, and $\operatorname{cog}$ nitive vision. He has published the textbook Pattern Recognition: A Statistical Approach (Englewood Cliffs, NJ, USA: PrenticeHall, 1982) and over 700 scientific articles.

Dr. Kittler also serves on the editorial board of several scientific journals in pattern recognition and computer vision. 